\title{
Metformin inhibits leptin-induced growth and migration of glioblastoma cells
}

\author{
RITA FERLA $^{1}$, EVA HASPINGER ${ }^{1,2}$ and EVA SURMACZ ${ }^{1}$ \\ ${ }^{1}$ Sbarro Institute for Cancer Research and Molecular Medicine, Temple University, Philadelphia, PA 19122, USA; \\ ${ }^{2}$ Department of Medical Oncology, University of Verona, 37189 Verona, Italy
}

Received April 4, 2012; Accepted July 18, 2012

DOI: $10.3892 / \mathrm{ol} .2012 .843$

\begin{abstract}
Metformin, a derivative of biguanide, is a first-line therapy for type 2 diabetes mellitus. Since the drug has been shown to significantly reduce the risk of various cancers and cancer mortality in diabetic patients, it is being considered as a potential anticancer therapeutic or preventive agent. In cellular models, metformin inhibits the growth of many types of cancer cells; however, its effects on glioblastoma multiforme $(\mathrm{GBM})$ are not well characterized. Here, we analyzed the effects of metformin on the growth and migration of LN18 and LN229 GBM cells cultured under basal conditions or exposed to leptin, a cytokine that has recently been implicated in GBM development. We found that 2-16 $\mathrm{mM}$ metformin reduced basal and leptin-stimulated growth of GBM cells in a dose-dependent manner. Furthermore, the drug significantly inhibited the migration of GBM cells. The action of metformin was mediated through the upregulation of its main signaling molecule, the adenosine monophosphate-activated protein kinase (AMPK), as well as through the downregulation of the signal transducer and activator of transcription 3 (STAT3) and the $\mathrm{Akt} / \mathrm{PKB}$ serine/threonine protein kinase. In leptin-treated cells, the drug reversed the effects of the cytokine on the AMPK and STAT3 pathways, but modulated Akt activity in a cell-dependent manner. Our results suggest that metformin or similar AMPK-targeting agents with optimized bloodbrain-barrier penetrability could be developed as potential treatments of GBM and could be used in conjunction with other target drugs such as leptin receptor antagonists.
\end{abstract}

\section{Introduction}

Metformin, a biguanide derivative, is a first-line oral medication for type 2 diabetes mellitus. The best known effects of

Correspondence to: Professor Eva Surmacz, Sbarro Institute for Cancer Research and Molecular Medicine, Temple University, Bio Life Building, Rm. 425, 1900 N. 12th Street, Philadelphia, PA 19122 , USA

E-mail: surmacz@temple.edu

Key words: metformin, leptin, glioblastoma, AMPK, proliferation, migration metformin include the suppression of hepatic glucose production and reduction of insulin resistance in peripheral tissues (1). In addition, the drug enhances both peripheral glucose uptake and fatty acid oxidation (1). The Diabetes Prevention Trial demonstrated that metformin decreased the incidence of diabetes development in at risk populations by $30 \%$ (2).

The key mechanism of metformin action is the activation of its major effector molecule, the adenosine monophosphateactivated protein kinase (AMPK), a sensor of cellular energy that is normally activated under conditions of starvation (3-5). The upregulation of AMPK inhibits several anabolic/mitogenic pathways activated by growth factors and nutrients, including the phosphatidylinositol-3-kinase (PI-3K) pathway, the mammalian target of rapamycin (mTOR) and extracellular signal-regulated kinases 1 and 2 (ERK1/2) (6). Further downstream, the consequences of AMPK stimulation may include the inhibition of the cell cycle regulator cyclin D1, the downregulation of critical transcriptional regulators such as hypoxia inducible factor $1 \alpha$, nuclear factor $\kappa \mathrm{B}$ and the c-myc protein, and depletion of other mitosis-related proteins (7). On a cellular level, in addition to its insulin-sensitizing effects, the drug is known to inhibit cell growth, migration, invasion and angiogenesis (7).

The above effects are particularly attractive in the context of the potential use of metformin in restricting premalignant or malignant cell growth. Indeed, in cellular models, metformin inhibited the growth of breast, colorectal, pancreatic, lung, ovarian and prostate cancer cells (7). The drug also suppressed the expression of human epidermal growth factor receptor 2 (HER2) in certain breast cancer cell lines. In animal models, metformin reduced chemically-induced carcinogenesis in various organs (mammary gland, intestine, endometrium, skin, lung and pancreas), and inhibited the growth of breast cancer xenografts and mammary tumors in HER2 transgenic mice (7-12).

Epidemiological studies in diabetic patients have suggested that metformin significantly reduces the risk of pancreatic, liver, colorectal, breast, endometrial and bladder cancer development, and decreases cancer-related mortality (7). Moreover, the drug improves the response to neoadjuvant breast cancer therapy in diabetic women (7). At present, several prospective clinical trials are evaluating metformin as a single or combined treatment for solid tumors (7) and demonstrate its potential value for cancer prevention (13) (www.clinicaltrials.gov).

Studies in vivo demonstrated that metformin can, at least to some extent, cross the blood-brain barrier (BBB) through 
an organic cation transporter-dependent mechanism and exert pharmacological effects, including AMPK activation, in intact brain $(8,14)$ and glioma cells in vitro (4). Notably, the AMPK pathway appears to be critical for the growth of epidermal growth factor receptor-dependent glioblastoma multiforme (GBM), and the activation of AMPK by its agonist significantly reduces GBM proliferation (15). However, only a few studies addressed the effects of the leading AMPK-inducing pharmaceutical agent, metformin, on brain tumor biology. The results suggest that the drug reduces the growth and/or migration of different rat or human glioma cell lines that have a mutation in the phosphatase and tensin homolog (PTEN) gene and lack expression of the PTEN tumor suppressor protein $(4,16,17)$.

Here, we analyzed the effects of metformin on basal and leptin-induced growth and migration of PTEN-positive LN18 and LN229 GBM cell lines. Leptin is a multifunctional cytokine that has been shown to regulate metabolic and neoplastic activities in many cell types $(18,19)$. We reported previously that leptin and its receptor (ObR) are overexpressed in different human brain tumors and that their levels correlate with the degree of malignancy, being the most abundant in GBM (20). In ObR-positive LN18 and LN229 cells, leptin acts as a mitogen/survival factor and its effects coincide with the stimulation of the PI-3K/Akt, signal transducer and activator of transcription 3 (STAT3) pathways as well as the modulation of ERK1/2 signaling and retinoblastoma protein $(\mathrm{pRb})$ phosphorylation (20).

\section{Materials and methods}

Cell lines and growth conditions. ObR-positive LN18 and LN229 glioblastoma cell lines were obtained from ATCC (Manassas, VA, USA). Both cell lines were cultured in low-glucose Dulbecco's modified Eagle's medium (DMEM) (Cellgro Mediatech, Manassas, VA, USA) supplemented with $5 \%$ fetal bovine serum (Cellgro Mediatech) as described in a previous study (20). The study was approved by the Biosafety Committee at Temple University, PA, USA.

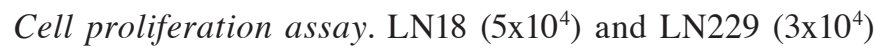
cells were seeded in 24-well plates in growth medium. After $24 \mathrm{~h}$, the cells were placed in serum-free medium (SFM; high-glucose DMEM supplemented with $0.42 \mathrm{~g} / \mathrm{ml}$ bovine serum albumin, $1 \mu \mathrm{M} \mathrm{FeSO}_{4}$ and $2 \mathrm{mM} \mathrm{L}$-glutamine) for a further $24 \mathrm{~h}$. Next, the cells were treated for $48 \mathrm{~h}$ with 2, 4, 8 and $16 \mathrm{mM}$ metformin (Sigma Aldrich, St. Louis, MO, USA) in the presence or absence of $200 \mathrm{ng} / \mathrm{ml}$ leptin, or were left untreated. At the conclusion of the experiment, the cells were counted under the microscope using the trypan-blue exclusion method. Each experiment was repeated a minimum of 3 times.

Cell migration assay. LN18 and LN229 cells (1.5x10 4 cells) suspended in modified SFM (high-glucose DMEM supplemented with $0.42 \mathrm{~g} / \mathrm{ml}$ bovine serum albumin, $1 \mu \mathrm{M} \mathrm{FeSO}_{4}$ and $2 \mathrm{mM}$ L-glutamine, and 0.5 and 1\% of FBS for LN229 and LN18, respectively) were seeded in the upper chambers of a Transwell system (24-well format, polycarbonate filters, $8-\mu \mathrm{M}$ pore size, Corning, Costar, NY, USA). The medium in the lower chambers was supplemented with leptin (200 ng/ml) acting as a chemoattractant, whereas metformin $(8$ and $16 \mathrm{mM})$ was added in the upper chambers and the cells were incubated for $16 \mathrm{~h}$. Subsequently, the cells on the upper surface of the filters were removed, while the cells that migrated to the underside of the filters were stained with Giemsa stain for $20 \mathrm{~min}$ and counted ( 5 fields/well) using a contrast phase microscope (Olympus CKX FA), at magnification x10. The average number of cells/field was determined \pm SD. Each experiment was repeated a minimum of 3 times.

Western blot (WB) analysis. LN18 and LN229 at a concentration of $1.2 \times 10^{6}$ and $1.0 \times 10^{6}$ cells $/ 100 \mathrm{~mm}$ plate, respectively, were grown for $16 \mathrm{~h}$ and then placed in SFM for $24 \mathrm{~h}$. The cells were subsequently pretreated with $16 \mathrm{mM}$ metformin for $24 \mathrm{~h}$ then exposed to $200 \mathrm{ng} / \mathrm{ml}$ leptin for 30 and $60 \mathrm{~min}$. Untreated cells were used as controls. The cells were then lysed in 1\% NP40, $50 \mathrm{mM}$ HEPES pH 7.5, $250 \mathrm{mM} \mathrm{NaCl}$, $5 \mathrm{mM}$ EDTA pH 8.0, 0.1\% SDS, $1 \mathrm{X}$ protease inhibitors (Complete Mini EDTA-free protease inhibitors, Hoffmann-La Roche, Nutley, NJ, USA) and phosphatase inhibitors (10 mM $\mathrm{Na}_{3} \mathrm{VO}_{4}$ and $50 \mathrm{mM} \mathrm{NaF}$ ). The expression of proteins was analyzed in $100 \mathrm{mg}$ total cell lysates. The following antibodies $(\mathrm{Ab})$ from Cell Signaling Technology (Danvers, MA, USA) were used for WB: for phospho-AMPK $\alpha$, AMPK $\alpha$ Thr172 D79.5E monoclonal (m)Ab 1:1000; total AMPK $\alpha$, AMPK $\alpha$ polyclonal (p)Ab 1:1000; phospho-Akt, Akt Ser473 pAb, 1:1000; total Akt, Akt pAb, 1:1000; phospho-STAT3, STAT3 Tyr705, D3A7 mAb, 1:1000; and for total STAT3, STAT3 79D7 mAb, 1:1000. The glyceraldehyde-3-phosphate dehydrogenase 6C5 (GAPDH) Ab 1:1000 was obtained from Santa Cruz Biotechnology, CA, USA. The intensity of WB bands corresponding to studied proteins was analyzed by the Image J 1.44 program.

Statistical analysis. The results of the growth and migration experiments were analyzed by a two-tailed distribution paired Student's t-test. P-values $\leq 0.05$ were considered to indicate a statistically significant result.

\section{Results}

Leptin stimulates the growth of GBM cells, while metformin counteracts this effect. The effects of metformin in PTENpositive, leptin-responsive brain cancer cells have never been examined. We assessed how the drug affects basal and leptindependent proliferation of LN229 and LN18 cells (Fig. 1). We found that metformin at $2-16 \mathrm{mM}$ significantly decreased normal cell growth in both cell lines relative to untreated cells. The activity of the drug was dose-dependent and the best cytostatic result was observed with $16 \mathrm{mM}$, while lower concentrations produced lesser effects. Notably, LN18 cells appeared to be more sensitive to metformin than LN229 cells (Fig. 1).

Next, we evaluated the effects of 8 and $16 \mathrm{mM}$ metformin on leptin-induced proliferation of LN229 and LN18 cells (Fig. 2). In both cell lines, leptin increased cell growth by 25 and $20 \%$, respectively, whereas metformin counteracted these effects. In particular, in LN229 cells, 8 and $16 \mathrm{mM}$ metformin reduced cell proliferation to basal levels. In LN18 cells, both concentrations inhibited the growth to levels significantly below basal, suggesting that some activity of the drug is mediated through a leptin-independent mechanism (Fig. 2). 
LN229

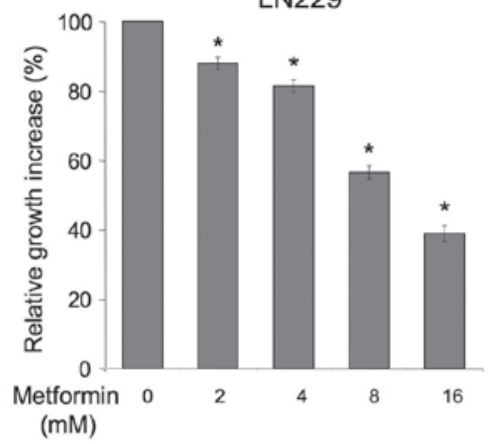

LN18

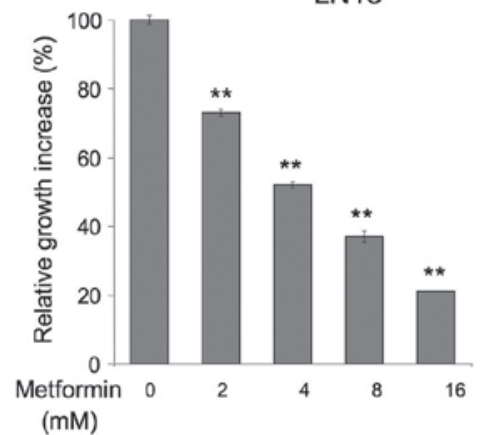

Figure 1. Effects of metformin on the normal growth of glioblastoma multiforme (GBM) cells. Cell growth in the presence or absence of 0-16 mM concentrations of metformin was assessed as described in Materials and methods. The number of cells in untreated cultures is taken as $100 \%$. Differences between untreated and treated cells $( \pm \mathrm{SE})$ were calculated as described in Materials and methods; ${ }^{*} \mathrm{p} \leq 0.05 ;{ }^{* *} \mathrm{p} \leq 0.01$.
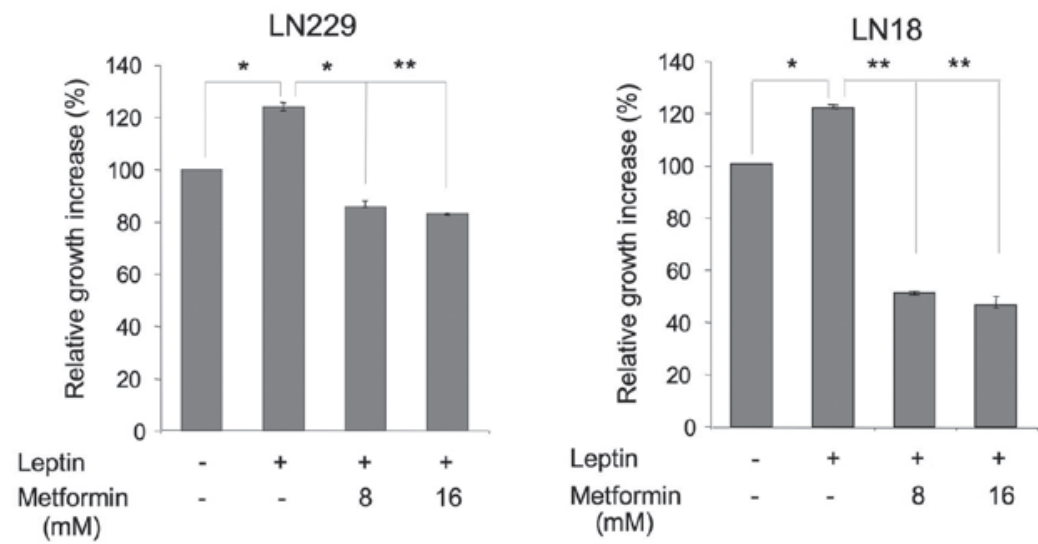

Figure 2. Effects of metformin on the leptin-dependent growth of glioblastoma multiforme (GBM) cells. Cell growth in the presence or absence of $200 \mathrm{ng} / \mathrm{ml}$ leptin and/or 8 or $16 \mathrm{mM}$ metformin was assessed as described in Materials and methods. The number of cells in untreated cultures is taken as $100 \%$. Differences between untreated and leptin-treated cells, and leptin-treated vs. leptin plus metformin $( \pm$ SE) were calculated as described in Materials and methods and are indicated by connecting lines; ${ }^{*} \mathrm{p} \leq 0.05 ; * * \mathrm{p} \leq 0.01$.
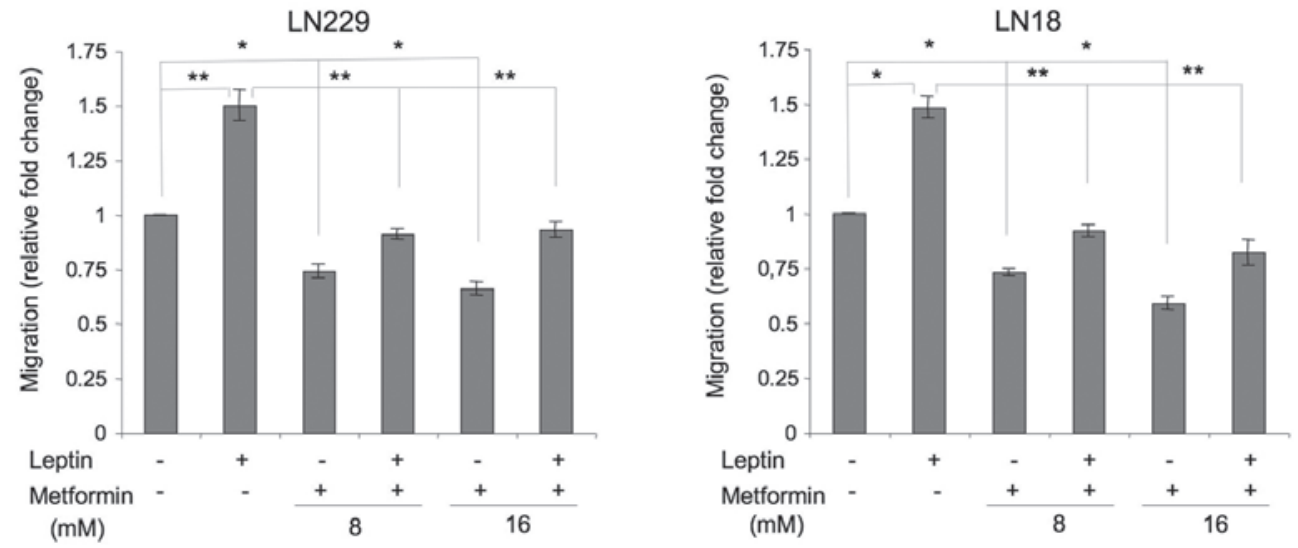

Figure 3. Effects of leptin and metformin on glioblastoma multiforme (GBM) cell migration. Cell migration in the presence or absence of $200 \mathrm{ng} / \mathrm{ml}$ leptin and/or 8 or $16 \mathrm{mM}$ metformin was assessed as described in Materials and methods. The number of cells in untreated cultures is taken as $100 \%$. Differences between untreated and leptin-treated or metformin-treated cells, and leptin-treated vs. leptin plus metformin $( \pm \mathrm{SE})$ were calculated as described in Materials and methods and are indicated by connecting lines; ${ }^{*} \mathrm{p} \leq 0.05 ;{ }^{* *} \mathrm{p} \leq 0.01$.

Leptin stimulates the migration of GBM cells, while metformin counteracts this effect. Leptin is a recognized motogenic and angiogenic factor in cancer models (21) and induces migration in rat glioma cells (22); however, its role in human GBM migration has never been studied. We found that in LN229 and LN18 cells, leptin increased cell migration by $\sim 50 \%$ (Fig. 3). In the presence of metformin, leptin-dependent migration was reduced to basal or slightly below basal levels (Fig. 3). 

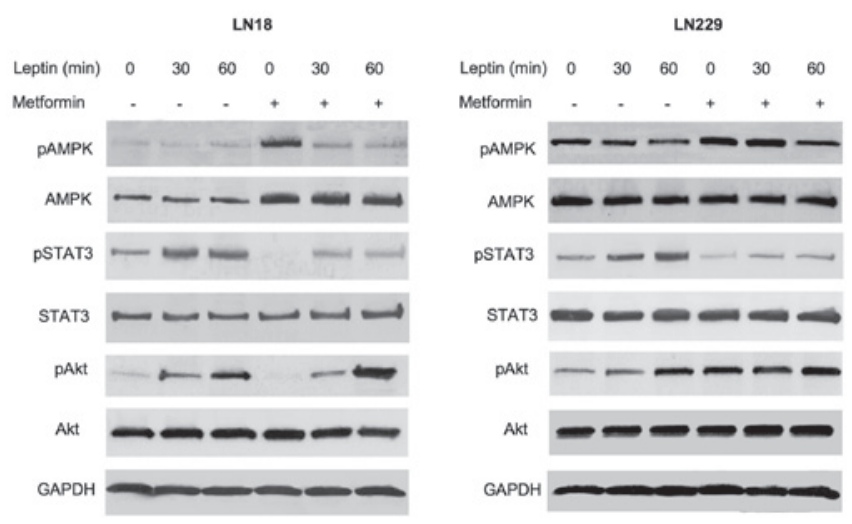

Figure 4. Effects of leptin and metformin on intracellular signaling in glioblastoma multiforme cells. The cells were pretreated or not with $16 \mathrm{mM}$ metformin and stimulated or not with $200 \mathrm{ng} / \mathrm{ml}$ leptin for 0-60 min, as described in Materials and methods. The levels of phosphorylated (p) and total AMPK, STAT3, and Akt proteins, and control protein GAPDH were assessed by western blot analysis using specific antibodies, as detailed in Materials and methods.

In addition, we observed that metformin alone significantly suppressed basal cell migration. In LN229 cells, the drug at 8 and $16 \mathrm{mM}$ inhibited basal cell migration by 26 and $34 \%$, respectively. In LN18 cells, the decrease of cell migration observed in the presence of metformin 8 and $16 \mathrm{mM}$ was 27 and $41 \%$, relative to the control (Fig. 3).

Effects of metformin on leptin signaling pathways in GBM cells. We previously demonstrated that leptin activates the STAT3 and Akt pathways, and downregulates ERK1/2 signaling in ObR-positive GBM cells (20). In addition, leptin is known to modulate AMPK in a cell context-dependent manner $(19,23)$, although its effects on this enzyme in brain tumor cells have never been studied. Here, we assessed how metformin modulates several signaling pathways in GBM cells cultured in the presence or absence of leptin (Fig. 4).

In LN18 cells, the basal levels of activated AMPK were low and leptin treatment did not modify its phosphorylation status. However, metformin pretreatment increased total AMPK levels by $\sim 90 \%$. The addition of leptin to metformintreated cultures did not modulate AMPK abundance, but it decreased AMPK phosphorylation by $90-120 \%$ (Fig. 4). In LN229 cells, leptin treatment for 30 or 60 min reduced the levels of phosphorylated AMPK by $\sim 40$ and $60 \%$, respectively, without affecting the basal expression of the enzyme. Metformin did not increase total AMPK levels in LN229 cells, but it moderately ( 40\%) elevated AMPK phosphorylation. The latter effect was effectively counteracted by leptin treatment, particularly at $60 \mathrm{~min}$ (Fig. 4).

In both cell lines, neither metformin nor leptin affected total STAT3 levels. Leptin treatment induced STAT3 phosphorylation at 30 and $60 \mathrm{~min}$ by $\sim 80-200 \%$, while metformin pretreatment reduced STAT3 activation to below basal levels. This effect of metformin was partially reversed by leptin addition (Fig. 4).

In LN18 and LN229 cells, leptin significantly stimulated Akt phosphorylation, particularly at $60 \mathrm{~min}$. Notably, metformin produced differential effects of Akt signaling in our cell models. In LN18 cells, metformin inhibited basal Akt phosphorylation below basal levels, but this effect was totally negated by leptin treatment. By contrast, in LN229 cells, metformin increased Akt phosphorylation by $250 \%$, and the addition of leptin did not significantly alter this response. Total Akt levels were not modified by either leptin or metformin treatment (Fig. 4).

\section{Discussion}

There is significant experimental evidence showing that cellular mechanisms controlling the metabolism converge with those implicated in the development and progression of neoplastic diseases (7). In particular, energy excess appears to act as a tumor promoter in the course of many common cancers (7). AMPK is a critical pathway regulating cellular response to energy imbalance and has also been shown to control cancer progression (24). A well-characterized and extensively used pharmaceutical agent that activates AMPK, thereby mimicking a state of energy depletion, is metformin. Metformin has proven efficacy in the treatment of diabetes and other metabolic diseases $(1,25)$. The drug has also shown value in diabetes prevention, and its potential in the treatment and prevention of cancer is currently being evaluated (7-11).

In this study, we analyzed the effects of metformin on the growth and migration of human GBM cells cultured either under basal conditions or exposed to leptin, a hormone known to regulate various metabolic, mitogenic and motogenic functions in normal and neoplastic cells (21). The results of this study may be summarized as follows: i) metformin restricts basal and leptin-stimulated growth of LN18 and LN229 GBM cells; ii) metformin inhibits basal and leptin-induced migration of LN18 and LN229 cells; iii) the action of metformin in these cells is mediated through the AMPK, STAT3 and Akt pathways; iv) metformin counteracts leptin effects on the AMPK and STAT3 pathways, but modulates Akt status in a cell-dependent manner.

This study confirmed our previous findings that leptin stimulates several growth-related intracellular pathways and acts as a mitogen in GBM cells (20). Furthermore, we demonstrated for the first time that leptin activates migration in human GBM cells, which is consistent with the observation that the hormone induces migration of rat C6 glioma cells (22).

In human LN18 and LN229 cells, metformin inhibited motogenic as well as mitogenic leptin activity. These findings are original as no prior study has addressed metformin interference with leptin activity in cancer models.

Significantly, we observed that metformin not only inhibited cell growth, migration and signaling induced by leptin but also restricted cell functions in the absence of the hormone. This suggests that the drug suppresses the constitutive activation of several mitogenic and motogenic pathways in GBM cells, which are frequently induced due to either lack of tumor suppressors, such as PTEN, or overexpression of activated oncogenic proteins e.g., the activated epidermal growth factor receptor mutant EGFRVIII, the insulin-like growth factor receptor, HER2, or focal adhesion kinase (FAK) (26-30). In fact, previous studies demonstrated that metformin inhibits growth and/or migration of PTEN-deficient rat or human glioma cell lines $(4,16,17)$. 
Notably, despite the differential genetic backgrounds of our PTEN-positive cell models (LN18: EGFR+++, HER2+, IGF-IR+, FAK++; LN229: EGFR+, HER2+++, EGFRvIII+, IGF-IR+++, FAK+), metformin activated AMPK in both cell lines, either through increased levels or elevated phosphorylation of the enzyme. This is consistent with the original observation of Guo et al that the proliferation of at least some GBM cells is significantly suppressed by AMPK activation $(15,27)$. Metformin also inhibited STAT3 activation in both our cell models, which confirms the importance of STAT3 signaling in GBM (31).

In contrast, we observed differential effects of metformin on Akt in LN18 and LN229 cells. In LN18 cells, the drug reduced basal and leptin-induced Akt phosphorylation, which confirms reports of metformin activity in other cancer models $(32,33)$. Conversely, in LN229 cells, metformin significantly increased basal Akt phosphorylation, and this process was not affected by leptin treatment. The reason for this difference is unclear, but it may be related to cell-specific upregulation of Akt by chronic metformin treatment, as noted in certain models $(34,35)$.

In summary, our results suggest that metformin or similar AMPK-targeting agents with optimized BBB penetrability could be developed as potential treatments of GBM. Such modalities could be used in conjunction with other target drugs, for example those which inhibit angiogenic and mitogenic pathways stimulated by leptin or other cytokines/growth factors. However, the analysis of metformin interaction with conventional anti-neoplastic treatments is necessary as the drug may decrease the efficacy of some chemotherapeutic agents (4).

\section{Acknowledgements}

This study was supported by funds from the Sbarro Health Research Organization, PA, USA.

\section{References}

1. Bosi E: Metformin - the gold standard in type 2 diabetes: what does the evidence tell us? Diabetes Obes Metab 11 Suppl 2: 3-8, 2009.

2. Ramachandran A and Snehalatha C: Diabetes prevention programs. Med Clin North Am 95: 353-372, viii, 2011.

3. Boyle JG, Salt IP and McKay GA: Metformin action on AMP-activated protein kinase: a translational research approach to understanding a potential new therapeutic target. Diabet Med 27: 1097-1106, 2010.

4. Janjetovic K, Vucicevic L, Misirkic M, et al: Metformin reduces cisplatin-mediated apoptotic death of cancer cells through AMPK-independent activation of Akt. Eur J Pharmacol 651: 41-50, 2011.

5. Miller RA and Birnbaum MJ: An energetic tale of AMPKindependent effects of metformin. J Clin Invest 120: 2267-2270, 2010.

6. Hardie DG: The AMP-activated protein kinase pathway - new players upstream and downstream. J Cell Sci 117: 5479-5487, 2004.

7. Jalving M, Gietema JA, Lefrandt JD, et al: Metformin: taking away the candy for cancer? Eur J Cancer 46: 2369-2380, 2010.

8. Anisimov VN: Metformin for aging and cancer prevention. Aging (Albany NY) 2: 760-774, 2010.

9. Ben Sahra I, Le Marchand-Brustel Y, Tanti JF and Bost F: Metformin in cancer therapy: a new perspective for an old antidiabetic drug? Mol Cancer Ther 9: 1092-1099, 2010.

10. Gonzalez-Angulo AM and Meric-Bernstam F: Metformin: a therapeutic opportunity in breast cancer. Clin Cancer Res 16: 1695-1700, 2010.

11. Kourelis TV and Siegel RD: Metformin and cancer: new applications for an old drug. Med Oncol 29: 1314-1327, 2012.
12. Pollak M: Metformin and other biguanides in oncology: advancing the research agenda. Cancer Prev Res (Phila) 3: 1060-1065, 2010.

13. Hosono K, Endo H, Takahashi H, et al: Metformin suppresses colorectal aberrant crypt foci in a short-term clinical trial. Cancer Prev Res (Phila) 3: 1077-1083, 2010.

14. Eyal S, Hsiao P and Unadkat JD: Drug interactions at the bloodbrain barrier: fact or fantasy? Pharmacol Ther 123: 80-104, 2009.

15. Guo D, Cloughesy TF, Radu CG and Mischel PS: AMPK: A metabolic checkpoint that regulates the growth of EGFR activated glioblastomas. Cell Cycle 9: 211-212, 2010.

16. Isakovic A, Harhaji L, Stevanovic D, et al: Dual antiglioma action of metformin: cell cycle arrest and mitochondria-dependent apoptosis. Cell Mol Life Sci 64: 1290-1302, 2007.

17. Beckner ME, Gobbel GT, Abounader R, et al: Glycolytic glioma cells with active glycogen synthase are sensitive to PTEN and inhibitors of PI3K and gluconeogenesis. Lab Invest 85: 1457-1470, 2005.

18. Zhang F, Chen Y, Heiman M and Dimarchi R: Leptin: structure, function and biology. Vitam Horm 71: 345-372, 2005.

19. Scolaro L, Cassone, M, Kolaczynski, JW, Otvos L Jr and Surmacz E: Leptin-based therapeutics. Expert Rev Endocrinol Metab 5: 875-889, 2010

20. Riolfi M, Ferla R, Del Valle L, et al: Leptin and its receptor are overexpressed in brain tumors and correlate with the degree of malignancy. Brain Pathol 20: 481-489, 2010.

21. Garofalo C and Surmacz E: Leptin and cancer. J Cell Physiol 207: 12-22, 2006.

22. Yeh WL, Lu DY, Lee MJ and Fu WM: Leptin induces migration and invasion of glioma cells through MMP-13 production. Glia 57: 454-464, 2009.

23. Hardie DG: AMPK: a key regulator of energy balance in the single cell and the whole organism. Int J Obes (Lond) 32 (Suppl 4): S7-S12, 2008.

24. Luo Z, Zang $M$ and Guo W: AMPK as a metabolic tumor suppressor: control of metabolism and cell growth. Future Oncol 6: 457-470, 2010.

25. Tang T, Lord JM, Norman RJ, Yasmin E and Balen AH: Insulinsensitising drugs (metformin, rosiglitazone, pioglitazone, D-chiro-inositol) for women with polycystic ovary syndrome, oligo amenorrhoea and subfertility. Cochrane Database Syst Rev CD003053, 2009.

26. Berezowska S, Diermeier-Daucher S, Brockhoff G, et al: Effect of additional inhibition of human epidermal growth factor receptor 2 with the bispecific tyrosine kinase inhibitor AEE788 on the resistance to specific EGFR inhibition in glioma cells. Int J Mol Med 26: 713-721, 2010.

27. Guo D, Hildebrandt IJ, Prins RM, et al: The AMPK agonist AICAR inhibits the growth of EGFRvIII-expressing glioblastomas by inhibiting lipogenesis. Proc Natl Acad Sci USA 106: 12932-12937, 2009.

28. Ang C, Guiot MC, Ramanakumar AV, Roberge D and Kavan P: Clinical significance of molecular biomarkers in glioblastoma. Can J Neurol Sci 37: 625-630, 2010.

29. Liu TJ, LaFortune T, Honda T, et al: Inhibition of both focal adhesion kinase and insulin-like growth factor-I receptor kinase suppresses glioma proliferation in vitro and in vivo. Mol Cancer Ther 6: 1357-1367, 2007.

30. Schlegel J, Piontek G, Budde B, Neff F and Kraus A: The Akt/ protein kinase B-dependent anti-apoptotic pathway and the mitogen-activated protein kinase cascade are alternatively activated in human glioblastoma multiforme. Cancer Lett 158: 103-108, 2000.

31. de la Iglesia N, Puram SV and Bonni A: STAT3 regulation of glioblastoma pathogenesis. Curr Mol Med 9: 580-590, 2009.

32. Zakikhani M, Blouin MJ, Piura E and Pollak MN: Metformin and rapamycin have distinct effects on the AKT pathway and proliferation in breast cancer cells. Breast Cancer Res Treat 123: 271-279, 2010.

33. Alimova IN, Liu B, Fan Z, et al: Metformin inhibits breast cancer cell growth, colony formation and induces cell cycle arrest in vitro. Cell Cycle 8: 909-915, 2009.

34. Yang J and Holman GD: Long-term metformin treatment stimulates cardiomyocyte glucose transport through an AMP-activated protein kinase-dependent reduction in GLUT4 endocytosis. Endocrinology 147: 2728-2736, 2006.

35. Sonntag B, Gotte M, Wulfing P, Schuring AN, Kiesel L and Greb RR: Metformin alters insulin signaling and viability of human granulosa cells. Fertil Steril 84 Suppl 2: 1173-1179, 2005. 